\title{
Analysis of Bulging in Continuously Cast Slabs by Bending Theory of Continuous Beam*
}

\section{By Akihiko YOSHII** and Shigefumi KIHARA**}

\section{Synopsis}

The bending theory of continuous beam has been applied to the analysis of bulging in continuously cast slabs. A simple algorithm was formulated to transform a through-thickness distribution of creep strain in a shell into the shell curvature, and equations were given for the analysis model of continuous beam with multi-supports. Simulations have proved that the model satisfactorily predicts the variations of bulging profile with multi-roll arrangement and the transitional bulging including unsteady fluctuations.

\section{Introduction}

Many problems arise when the bulging analysis of continuously cast steel slabs is made by the conventional methods of structural analysis such as the finite element method. The calculation becomes very large in scale because the high creep rate near the solidus temperature requires a fine mesh dividing both in time and space to guarantee the stability or accuracy of numerical integrations. Furthermore, it is difficult to take into consideration the effect of moving body during the withdrawal of the cast slabs. Much ambiguity remains in spite of a number of attempts to analyze the bulging, by using simplified models based on the beam bending theory or models for mathematical formulation. ${ }^{1-3}$ )

The difficult problems are as follows.

1) Formulation of the non-uniformity of mechanical properties and non-linearity of stress (or creep strain) through the shell thickness in the beam modeling.

2) Determination of the boundary conditions at both ends (roll supporting points) and the initial value of strain (an initial curvature of beam) at the first roll in the conventional one roll pitch model.

As an approach to a solution of these problems the authors have formulated a simple algorithm to transform a micro-distribution of creep strain in the shell thickness direction into the shell curvature as a macroquantity and formulated equations for the analysis of continuous beam model with multi-roll supports. As the result, an improved efficient method was implemented in the bulging analysis including the transitional response. ${ }^{4)}$

\section{Modeling and Theory}

\section{Formulation for Beam Bending Theory}

Bulging of wide slabs can be analyzed by the model of two-dimensional structure as shown in Fig. 1. The strain distribution $\varepsilon(y)$ in the thickness direction is expressed as

$$
\varepsilon(y)=-\left(y-y_{0}\right) \rho
$$

where $\rho$ is the bending curvature induced by the bending moment $M$ on a slab section (see Fig. 2), and $y_{0}$ indicates the position of neutral axis.

The stress distribution $\sigma(y)$ is correlated with the strain distribution $\varepsilon(y)$ by

$$
\sigma(y)=E(y)\left\{\varepsilon(y)-\varepsilon_{\mathrm{e}}(y)\right\}
$$

where $\varepsilon_{\mathrm{c}}(y)$ and $E(y)$ denote creep strain and Young's modulus, respectively.

The bending moment in the beam which has a depth of $h$ is written as

$$
M=-\int_{0}^{h}\left(y-y_{0}\right) \sigma d y
$$

and the free axial force gives

$$
\int_{0}^{h} \sigma d y=0
$$

Substitution of Eqs. (1), (2) and (4) into Eq. (3) leads to the formulae as follows:

$$
M=D\left(\rho-\rho_{\mathrm{c}}\right)
$$

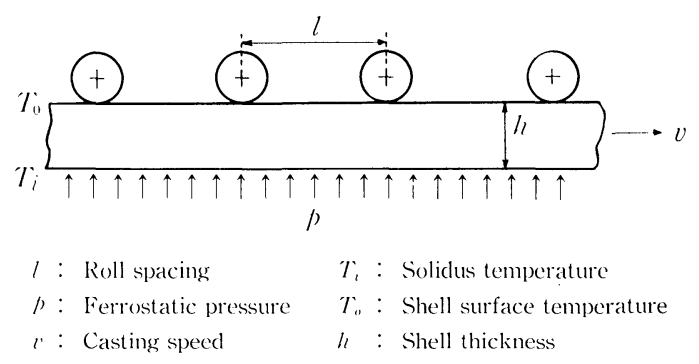

Fig. 1. Two-dimensional modeling and parameters used in the bulging analysis.

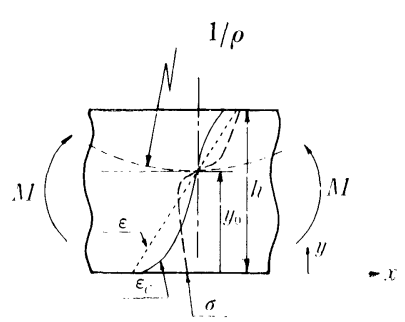

Fig. 2. Stress and strain distributions in the shell thickness direction of the model.

* Presented to the 110th ISIJ Meeting, October 1985, S1059, at Niigata University in Niigata. Manuscript received on February 10, 1986; accepted in the final form on June 13, 1986. (C) 1986 ISIJ

** Niihama Research Laboratory, Sumitomo Heavy Industries, Ltd., Sobiraki-cho, Niihama 792. 
where, $\quad \rho_{\mathrm{c}}=-\int_{0}^{h} y E \varepsilon_{\mathrm{c}} d y / D$

$$
D=\int_{0}^{h} E y^{2} d y-y_{0} \int_{0}^{h} E y d y
$$

and $y_{0}=\int_{0}^{h} E y d y / \int_{0}^{h} E d y+\int_{0}^{h} E \varepsilon_{\mathrm{c}} d y / \rho \int_{0}^{h} E d y$

Equation (6) represents the transformation of the micro-distribution $\varepsilon_{\mathrm{c}}$ in the thickness direction into the macro curvature $\rho_{\mathrm{c}}$ of the beam. Equation (5) is deduced to a bending equation of an elastic beam with flexural rigidity $D$ and initial curvature $\rho_{\mathrm{c}}$.

The second term of the right side of Eq. (8) has little effect on bulging in a practical condition, as is confirmed less than $5 \%$ by numerical simulations, and is neglected in the present study.

\section{Continuous Beam Model}

An ideal beam model shown in Fig. 3 is adequate for the analysis of bulging by a continuous beam model with multi-roll supports shown in Fig. 1.

Requirement of continuity of displacement at $n$-th roll and equilibrium of forces are given hereinafter. By assuming the pressure $p_{n}$ and flexural rigidity $D_{n}$ constant in the $n$-th roll spacing, the bending moment $M(x)$ and shearing force $F(x)$ in the interval are expressed by

$$
M(x)=M_{n-1}-F_{n-1}+\frac{1}{2} p_{n} x^{2}
$$

and

$$
F(x)=F_{n-1}-p_{n} x
$$

where $M_{n-1}$ and $F_{n-1}$ are the statically indeterminate moment and shearing force at $n-1$-th roll.

By substituting Eq. (9) into Eq. (5), the displacement $w(x)$ and its derivative $\theta(x) \quad(=d w / d x)$ are obtained by integrating the curvature $\rho\left(=d^{2} w / d x^{2}\right)$.

$$
\begin{aligned}
w(x)= & \frac{p_{n}}{24 D_{n}} x^{4}-\frac{F_{n-1}}{6 D_{n}} x^{3}+\frac{M_{n-1}}{2 D_{n}} x^{2}+\theta_{n-1} x \\
& +w_{n-1}+\int_{0}^{x} \int_{0}^{x} \rho_{\mathrm{c}} d x d x \quad \ldots \ldots \ldots \ldots \ldots \ldots \ldots \ldots \ldots \ldots \ldots \ldots \ldots \\
\theta(x)= & \frac{p_{n}}{6 D_{n}} x^{3}-\frac{F_{n-1}}{2 D_{n}} x^{2}+\frac{M_{n-1}}{D_{n}} x+\theta_{n-1}+\int_{0}^{x} \rho_{\mathrm{c}} d x
\end{aligned}
$$

Consequently, the following recurrent relations are obtained.

$$
\begin{aligned}
& -\frac{l_{n}^{3}}{6 D_{n}} F_{n-1}+\frac{l_{n}^{2}}{2 D_{n}} M_{n-1}+l_{n} \theta_{n-1}+w_{n-1}-w_{n} \\
& =-\int_{0}^{l_{n}} \int_{0}^{l_{n}} \rho_{\mathrm{c}} d x d x-\frac{p_{n}}{24 D_{n}} l_{n}^{4} \\
& -\frac{l_{n}^{2}}{2 D_{n}} F_{n-1}+\frac{l_{n}}{D_{n}} M_{n-1}+\theta_{n-1}-\theta_{n} \\
& =-\int_{0}^{l_{n}} \rho_{\mathrm{c}} d x-\frac{p_{n}}{6 D_{n}} l_{n}^{3} \\
& M_{n-1}-l_{n} F_{n-1}-M_{n}=-\frac{1}{2} p_{n} l_{n}^{2} \\
& F_{n-1}+k_{n} w_{n}-F_{n-1}=p_{n} l_{n}
\end{aligned}
$$

Equations (13) and (14) are the conditions of con-

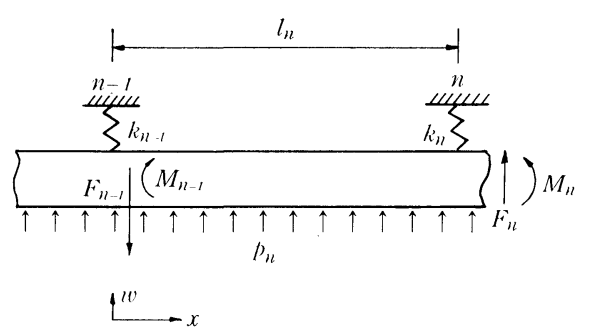

Fig. 3. Beam idealization for the formulations.

tinuity of $w$ and $\theta$ at $n$-th roll position, and Eqs. (15) and (16) are those of equilibrium of $M$ and $F$ in the interval.

When the number of roll spacings in the model is $\mathcal{N}$, simultaneous equations of $4 \mathcal{N}$ order are obtained, but the number of unknowns are $4 \mathcal{N}+4\left(w_{0}, \theta_{0}, M_{0}\right.$, $\left.F_{0}, \ldots, W_{n}, \theta_{n}, M_{n}, F_{n}, \ldots, w_{N}, \theta_{N}, M_{N}, F_{N}\right)$. Therefore, four boundary conditions in total at the both ends must be given to solve the equations according to the idealizations: e.g., both ends fixed, cyclic conditions, given force boundary, etc.

\section{Procedure of Numerical Calculations}

Based on the models mentioned above, bulging behavior is numerically calculated in the following procedures;

(1) The creep strain distribution, $\varepsilon_{\mathrm{c}}(x, y)$ in a slab at the time $t$, is transformed to $\rho_{\mathrm{c}}(x)$ by Eq. (6).

(2) The simultaneous equations are solved by calculating the creep dependent terms (the right terms) of Eqs. (13) and (14), to determine $M_{n}, F_{n}$, $w_{n}$, and $\theta_{n}(n=0,1, \ldots, \mathcal{N})$.

(3) The moment distribution $M(x)$ and bulging profile $w(x)$ are calculated from Eqs. (9) and (11).

(4) The stress distribution $\sigma(x, y)$ is obtained as

$$
\text { and } \quad \sigma=E\left(\varepsilon-\varepsilon_{\mathrm{c}}\right) \quad \text { from Eq. (2). }
$$

Young's modulus $E$ reported by Mizukami et al. ${ }^{5)}$ was used in the present calculations.

(5) The creep strain increment $\Delta \varepsilon_{\mathrm{c}}(x, y)$ in the time increment $\Delta t$, calculated by

$$
\Delta \varepsilon_{\mathrm{c}}=\frac{\sigma}{|\sigma|} \dot{\varepsilon}_{\mathrm{c}}\left(\sigma, \varepsilon_{\mathrm{c}}\right) \Delta t
$$

is added to $\varepsilon_{\mathrm{c}}(x, y)$. The creep rate $\dot{\varepsilon}_{\mathrm{c}}$ in a stress cycle is calculated according to the theory of strain hardening. ${ }^{\text {() }}$

(6) When a time step is synchronized with mesh moving, the quantities related to the creep strain $\varepsilon_{\mathrm{c}}\left(\right.$ or $\left.\rho_{\mathrm{c}}\right)$ are shifted by one mesh in $x$ direction. The values of the first mesh in $x$ direction is set to zero except for the cyclic condition.

By repeating the above procedure, the time history of $w(x), \sigma(x, y)$ and $\varepsilon(x, y)$ are obtained.

\section{Creep Law}

The primary creep is assumed to be dominant in the bulging, because the strain range is small enough 
$(<1 \%)$ and the cycle time of stress alternation with withdrawal speed is also short $(<30 \mathrm{sec})$. In the primary creep region the strain may be expressed by the formula

$$
\varepsilon_{\mathrm{c}}=A \exp [-\alpha /(T+273)] \sigma^{m} t^{n}
$$

with $\varepsilon_{\mathrm{c}}$ : creep strain, $\sigma$ : stress $\left(\mathrm{kgf} / \mathrm{mm}^{2}\right), t$ : time (sec), and $T$ : temperature $\left({ }^{\circ} \mathrm{G}\right)$.

The parameters $A, \alpha, m$ and $n$ have been studied by several authors, ${ }^{7-10}$ ) but the creep behavior compared is considerably different especialy when the data is extrapolated to high temperature region $\left(>1350^{\circ} \mathrm{C}\right)$. In the following calculations, $A, \alpha$ and $n$ are determined from the data of Fujii et al. ${ }^{7 \text { ) (for }}$ a middle carbon $\mathrm{Al}-\mathrm{Si}$ killed steel), which covers the highest temperature $\left(1350^{\circ} \mathrm{C}\right)$ so far reported.

Figure 4 shows time--strain curves corresponding to Eq. (18) used in the calculations. For the exponent $m$, Palmaers' data ${ }^{8)}$ is used to make up for the data in Ref. 7).

The strain-hardening theory ${ }^{6}$ ) is applied to express creep rate $\dot{\varepsilon}_{\mathrm{c}}$ (in Eq. (17)) in the shell subjected to alternating load (stress). According to the theory, $\dot{\varepsilon}_{\mathrm{c}}$ is derived from Eq. (18) as

$$
\dot{\varepsilon}_{\mathrm{e}}=n A^{1 / n} \exp [-\alpha / n /(T+273)] \sigma^{m / n} \varepsilon_{\mathrm{c}}^{(n-1) / n}
$$

In the present calculations, special considerations are given to avoid probable singurality or instability at $\varepsilon_{\mathrm{c}} \sim 0$.

\section{Influence of Boundary Condition}

In available reports about observed bulging, the rolls are arranged in an unequal spacing. ${ }^{11-13)}$ The bulging analyses by the continuous beam model give good agreement with these data and show that the arrangement of unequal pitch of roll has a large effect on the bulging.

An example of calculation is shown in Fig. 5, on the basis on Wünnenberg's measurement ${ }^{11)}$ by using the idealized model shown in Fig. 6. The solid line is for the continuous beam model under the measured condition, the broken line for the one pitch model with the fixed ends, and the chain line for the cyclic boundary condition.

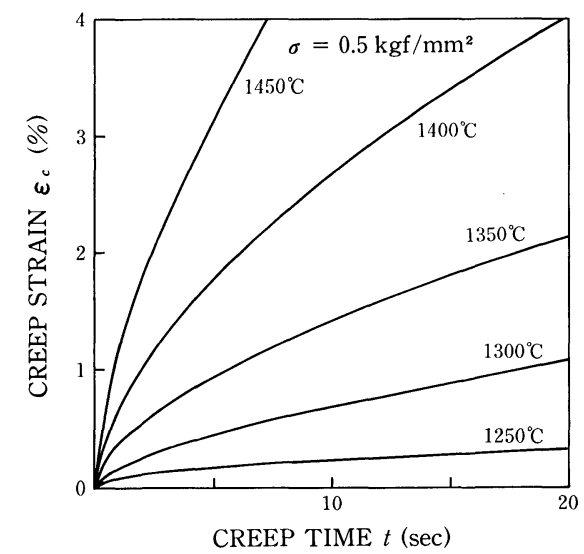

Fig. 4. Time-strain curves used for the analysis $(\sigma=0.5$ $\mathrm{kgf} / \mathrm{mm}^{2}$ ).
This shows that the differences due to the boundary conditions are obvious in a way that:

(1) The bulging under the measured condition is very different from the one under the cyclic boundary condition that is often used in the analysis.

(2) The strain at the roll point of down stream side is overestimated in the case of both ends fixed, which is also a popular assumption for the analysis.

\section{Unsteady or Transitional Bulging}

\section{Response of Strand Standstill}

It is well known that the bulging rapidly increases when the withdrawal is stopped. Figure 7 shows an example of a transitional bulging when the casting speed is changed stepwise from 1.8 to $0.85 \mathrm{~m} / \mathrm{min}$, then to 0 (stop). A rapid increase of the bulging during the strand standstill is shown. The figure shows that the time response in strand standstill is similar to the primary creep law $\left(\sim t^{n}\right)$ and coincident with the measured one. This means that the assumption that the bulging is mainly governed by the primary creep is valid. For comparison with the measured values the broken line shows a modification using the theoretical solution of rectangular plate, ${ }^{14)}$ by taking into account the finite width effect of slabs. The shell thickness increase during strand standstill is not considered in this calculation.

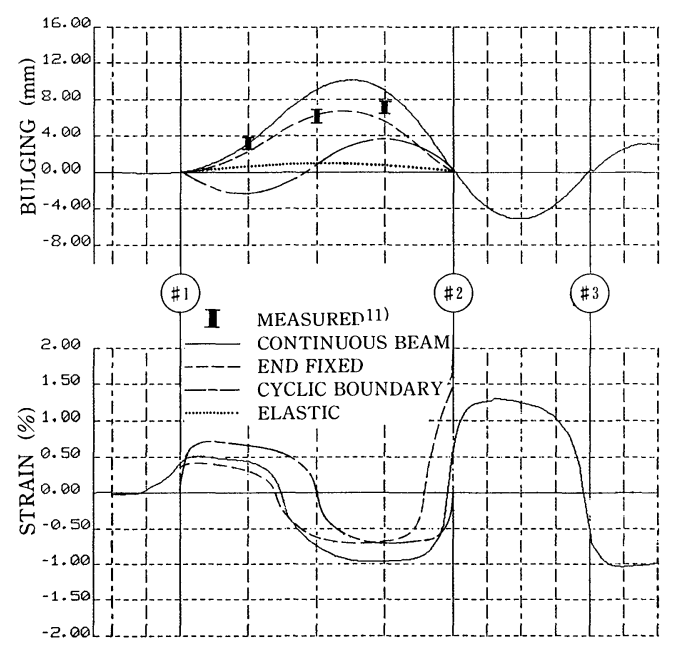

Fig. 5. The bulging and strain profiles and differences originated by the boundary conditions.

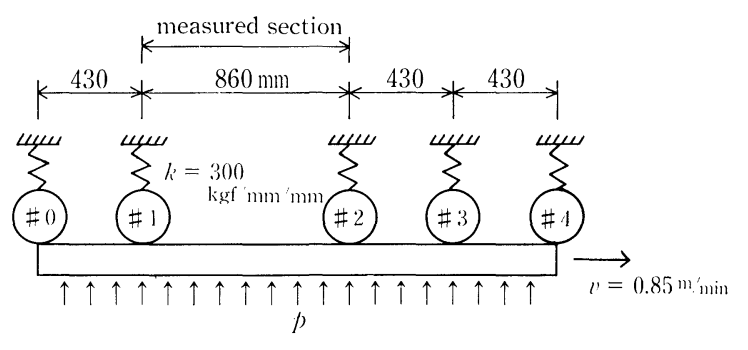

$\mathrm{T} i=1470^{\circ} \mathrm{C}, \quad T_{0}=1030^{\circ} \mathrm{C}, \quad p=0.027 \mathrm{kgf} / \mathrm{mm}^{2}, \quad h=79 \mathrm{~mm}$

Fig. 6. Parameters of the model analyzed. 


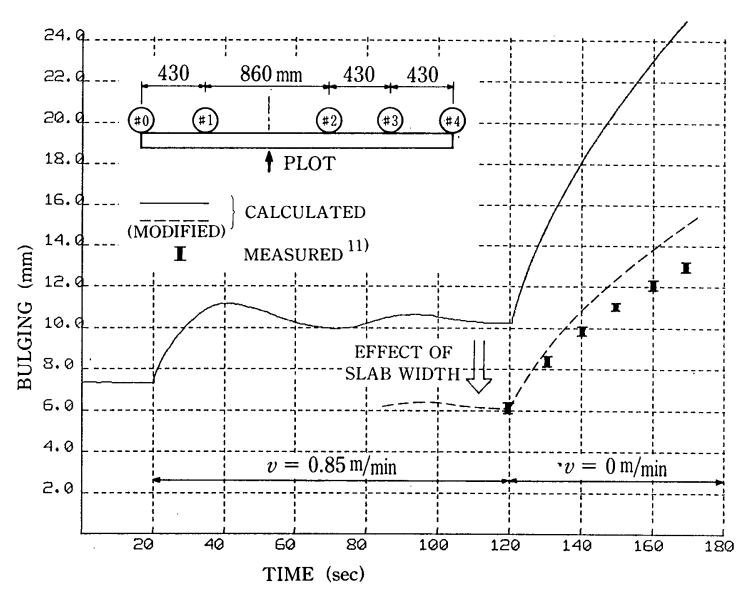

Fig. 7. The transitional bulging response when casting speed is changed stepwise from $1.8 \mathrm{~m} / \mathrm{min}$ to 0 (stop).

\section{Unsteady Bulging Accompanied by Speed Variation}

Figure 7 shows that oscillation of bulging arises when the speed is changed from 1.8 to $0.85 \mathrm{~m} / \mathrm{min}$. Such oscillation becomes more significant in amplitude and continues for longer time as the number of rolls is increased.

A similar tendency is observed in the unsteady bulging when a cyclic fluctuation is given to the casting speed. Figure 8 shows the unsteady behavior and profile of bulging at the time of maximum and minimum speed with fluctuation of $5 \%$ of the average casting speed. The amplitude of bulging fluctuations becomes larger in a down stream side. Thus, the unsteady bulging may become significant even by a small speed fluctuation by the effect of the continuation of the equal pitch rolls.

The model in Fig. 8 complies with the bench mark data submitted to the Joint Society on Iron and Steel Basic Research of ISIJ ${ }^{15}$ ) and this calculation was made by 8-roll model of equal pitch with the boundaries of fixed both ends.

\section{Conclusions}

The slab bulging analysis has become practical by formulating simple algorithms on continuous beam modeling which takes into account the stress and creep strain distribution in the shell thickness direction. Practical application of this model, including the elastic creep modeling, primary creep and strain hardening law in stress cycle, has proved to be valid in good coincidence with various measured data.

Various analyses by the present method have provided the following useful information.

(1) Bulging profiles largely depend on the arrangement of roll spacing. Consequently, a complicated bulging at the region where the roll spacing varies, can only be analyzed by the model of continuous beam with multi-supports.

(2) The rapid increase of bulging during strand standstill is similar to the primary creep response $\left(\sim t^{n}\right)$ and shows good coincidence with the measured data. This implies the validity of the assumption that bulging is mainly governed by the primary creep.
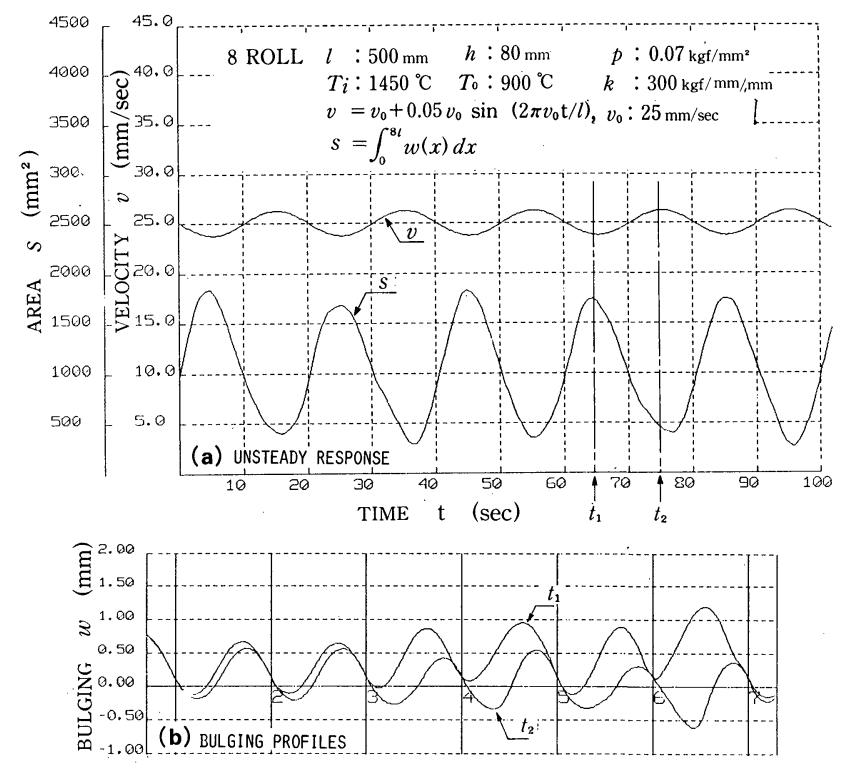

Fig. 8. An example of unsteady response and profiles of the bulging when a small speed fluctuation of $5 \%$ is given.

(3) Unsteady bulging arises when the casting speed varies or fluctuates. This becomes significant, as the number of rolls with a spacing of equal-pitch is increased.

\section{REFERENCES}

1) K. Miyazawa and K. Schwerdtfeger: Ironmaking and Steelmaking, 6 (1979), 68.

2) A. Palmaers, A. Atienne and J. Mignen: Stahl u. Eisen, 99 (1979), 1039.

3) T. Matsumiya, H. Kajioka and Y. Nakamura: Seitetsu Kenkyu, (1982), No. 310, 402.

4) A. Yoshii and S. Kihara: JSSC 9th Symposium on Computational Mechanics in Structural Engineering and Related Fields, Soc. Steel Construction Japan, Tokyo, (1985), 395.

5) H. Mizukami, K. Murakami and Y. Miyashita: Tetsu-toHagané, 63 (1977), S562.

6) Research and Development of Methods for Inelastic Structural Analysis, I, ed. by JSME Research Corporation SubCommittee 46, Japan Soc. Mech. Eng., Tokyo, (1977), 32.

7) H. Fujii, T. Ohashi, M. Oda, R. Arima and T. Hiromoto: Tetsu-to-Hagané, 67 (1981), 1172.

8) A. Palmaers: C.R.M., (1978), No. 53, 23.

9) T. Matsumiya: Tetsu-to-Hagané, 71 (1985), S1018.

10) J. Y. Lamant, M. Larrecq, J. P. Birat, J. L. Hensgen, J. D. Weber and J. C. Dhuyvetter: Continuous Casting '85, The Inst. Metals, London, (1985), 37.1.

11) K. Wünnenberg: Stahl u. Eisen, 98 (1978), 245.

12) Y. Sugitani, M. Nakamura and T. Kanazawa: Tetsu-toHagané, 70 (1984), S898; Trans. ISIJ, 25 (1985), B9.

13) S. Maeno, K. Wada, Y. Ito and H. Nagano: Tetsu-toHagané, 69 (1983), S938; Trans. ISIJ, 24 (1984), B82.

14) S. Timoshenko and S. Woinowsky-Krieger: Theory of Plates and Shells, 2nd ed., MaGraw-Hill, New York, (1959), 185.

15) Mechanics Related Behavior in Continuous Casting, ed. by Comm. on Mechanics Related Behavior in Continuous Casting, The Joint Soc. Iron Steel Basic Res., ISIJ, Tokyo, (1985). 94. 